\title{
Energy Efficient Protocol for Aggregation Head Selection in Wireless Sensor Network
}

\author{
Sarika Yadav and Rama Shankar Yadav
}

\begin{abstract}
Data aggregation mechanism is employed to reduce the redundant messages and energy consumption by collecting and aggregating messages from different sensor nodes in wireless sensor networks. We present an energy efficient data aggregation protocol for selection of aggregation head on the basis of its residual energy and nearness to the base station as well as comparative threshold energy when an event is detected. The efficiency of this aggregation protocol is shown through simulation results in terms of network lifetime by estimating number of dead and idle sensor nodes after fixed number of rounds as well as number of packets transmitted to base station as compared with the existing approaches.
\end{abstract}

Index Terms-Aggregation head, data aggregation, coverage, network lifetime.

\section{INTRODUCTION}

With advent of MEMS technology [1], wireless sensor networks have become a leading solution to enable data collection in important areas such as scientific studies, military and battlefield surveillance, monitoring industry manufacturing units, environmental monitoring, target tracking, assisted navigation etc. All of these applications depend on the ability of wireless sensor nodes to collect changes in physical attributes such as light, temperature, pressure, vibrations, humidity etc. as well as their ability to communicate with each other and base station [2]. It is infeasible for all the sensor nodes to transmit the sensed data to the base station in view of the fact that wireless sensor nodes are energy and distance constrained. Furthermore, data collected from neighboring sensor nodes is persistently redundant and highly interrelated. In such scenarios, sensor nodes can transmit data to a local collector or assigned head which combines data from all the neighboring sensor nodes residing in its connectivity and then transmits the concise packet to the base station. This is accomplished by data aggregation [3] that can be defined as the guiding principle to combine the sensed data from numerous sensor nodes with elimination of redundancy and provide collective information toward the base station. In this paper, we attempt to enhance the network lifetime by suggesting a protocol for selection of aggregation head on the basis of its residual energy and nearness to the base station as well as its threshold energy when an event is detected.

The review of literature is briefed in Section II whereas problem description is done in Section III. In Section IV, the proposed aggregation head $(\mathrm{AH})$ selection model is discussed

Manuscript received July 10, 2015; revised September 22, 2015.

The authors are with the Department of Computer Science and Engineering, Motilal Nehru National Institute of Technology, Allahabad, India (e-mail: sarikayash18@gmail.com, rsy@mnnit.ac.in). in detail. The performance evaluation of our proposal is given in Section V. Finally, conclusion and future work are given in Section VI.

\section{RELATED WORK}

With successful research contributions in previous decade, some foremost approaches are outlined in this section proposed for wireless structure- free networks [4] to reduce energy consumption and enhance the network lifetime in terms of network connectivity and its coverage range. The very first protocol Data-Aware Any cast and Randomized Waiting (DAA+RW) [5] was proposed by Fan et al. for event-driven data aggregation in a structure-free wireless sensor network with aim to reduce number of transmissions. To reduce the total number of transmissions, a randomized waiting scheme is set up so that each sensor node that has to report event data can start its transmission after a random waiting time. Priority is assigned to those sensor nodes having same event data to transmit or closer to base station for better aggregation efficiency. Chih-Min et al. presented a Structure-Free and Energy- Balanced Data Aggregation (SFEBDA) [6] protocol for wireless sensor networks consisting of two stages. In the first stage, as an event occurs, some sensor nodes are selected as primary aggregators (PA) and secondary aggregators (SA) depending on their positions and regarded as a pair (PA/SA pair) for the next step where the sensor node closer to base station is designated as the aggregator and the other one as forwarding node to achieve both the forwarding node selection and early aggregation. In the second stage, these aggregators send the collected packets back to the base station. Sardouk et al. proposed Multi-Agent Data Aggregation (MADA) [7] protocol that works spontaneously when a sensor node detects significant information; it asks its adjoining neighbors to participate in order to aggregate the most representative information to be send to the base station. Each sensor node decides to participate or not in the data aggregation process depending on relevance participation factor which is determined on the basis of various parameters such as importance of the information, its density, the criticality of its position and energy. The $\mathrm{AH}$ transmits the aggregated data to the base station in a single hop or multiple hops depending on the network configuration. Boughami et al. have proposed Energy Efficient Data Aggregation Algorithm (EEDAA) [8] in which the energy conservation is enhanced by the selection of aggregator node (called $\mathrm{AH}$ ) and the participant sensor nodes on basis of their residual energy. Once a sensor node is selected as $\mathrm{AH}$, it collects the data from all the sensor nodes who want to send data and aggregates the data before transmitting to the base station. In persuasion of these protocols and identifying the requirements [9], we propose an 
energy efficient protocol for data aggregation where wireless sensor networks are deployed for crisis management not having very large coverage area thereby sensor nodes communicating with base station directly via single hop communication.

\section{PROBLEM DESCRIPTION}

Consider a wireless structure-free sensor network with the base station at the center. We identified in earlier research that the selection of aggregation head was underprivileged as the criteria was either event detection [7] or residual energy [8]. When the aggregation head was selected on the basis of residual energy [8], it was not considered whether it has sufficient amount of energy to perform computations for aggregation and can transmit the aggregated data to the base station. In case of having insufficient amount of energy, such an aggregation head can result in network holes on grounds of disconnectivity and shrinkage of coverage area of the base station in the wireless sensor network. Taking this factor into account, we suggest an energy efficient protocol for selection of data aggregation head on the basis of its residual energy and nearness to the base station as well as comparative threshold energy when an event is detected.

\section{PROPOSED Model}

On event detection, a sensor node will send request message to its one-hop distance neighbors along with its residual energy to all its neighbor sensor nodes using AODV protocol only after estimating $E_{\min }$, the minimum amount of energy required to perform aggregation and transmission over certain distance. The neighbor sensor nodes that receive the request message will calculate their participation relevance $\left(R_{P}\right)$ and compare it with $R_{P \text { Threshold. }}$ If $\mathrm{R}$ is greater than $R_{P \text { Threshold }}$ then sensor node will participate in data collection and reply with their residual energy to the requesting sensor node. If the residual energy of the requesting sensor node is highest and its $E_{\min }$ is greater than $E_{\text {Residual }}$ then it is elected as the Aggregation Head otherwise among its neighbor sensor nodes, the sensor node which has the highest residual energy and whose $E_{\min }$ is greater than $E_{\text {Residual }}$ is assigned as the Aggregation Head. Once a sensor node is selected as Aggregation Head, it collects the data from all the sensor nodes who want to send data and aggregates the data before transmitting to the base station.

\section{A. Calculation of $E_{\text {min }}$}

The event detecting sensor node will send a request to its closest neighbors only after estimating its $E_{\text {min }}$ presuming following activities:

1) Energy consumed to send ' $\mathrm{k}$ ' aggregation requests, $E_{A g R q}$ $\times k$

2) Energy consumed in receiving ' $m$ ' aggregation responses, $E_{R} \times m$

3) Energy consumed in aggregation process, $E_{A g}$

4) Energy consumed in transmitting aggregated message over distance ' $\boldsymbol{d}$ ', $E_{T} \times \boldsymbol{d}$

$$
E_{\min }=E_{A g R q} \times k+E_{R} \times m+E_{A g}+E_{T} \times \boldsymbol{d}
$$

\section{B. Selection of Aggregation Head}

The Aggregation Head ( $\mathrm{AH}$ ) can be any node that detects an event and initiates the aggregation session. The $\mathrm{AH}$ consumes more energy than other sensor nodes participating in the aggregation session, hence it should be selected carefully so as to increase the lifetime of the network. The following parameters are used in selecting the $\mathrm{AH}$ -

1) Its residual energy $E_{\text {residual }}$

2) Its nearness to the base station

3) Its position in the network, whether critical or edge

4) Its identifier id.

The various symbols used and their description are summarized in Table I.

\section{TABLE I: TERMINOLOGY USED}

\begin{tabular}{|c|c|}
\hline Symbol & Description \\
\hline$E_{\min }$ & $\begin{array}{l}\text { minimum amount of energy required to perform } \\
\text { aggregation and transmission over certain distance. }\end{array}$ \\
\hline$R_{P}$ & participation relevance \\
\hline$R_{\text {PThreshold }}$ & Optimum value for participation relevance $(0.7)$ \\
\hline$E_{\text {Residual }}$ & Energy threshold for residual energy \\
\hline$E_{A g R q}$ & Energy required for sending aggregation request \\
\hline$E_{R}$ & Energy consumed in receiving message \\
\hline$E_{T}$ & Energy consumed in transmitting message \\
\hline$E_{A g}$ & Energy consumed in aggregating message \\
\hline$D$ & Distance between aggregation head and base station \\
\hline$S N$ & Sensor Node \\
\hline$A H$ & Aggregation Head \\
\hline$B S$ & Base Station \\
\hline$E_{\text {initial }}$ & Energy at initial \\
\hline$E_{\text {residual }}$ & Energy left \\
\hline$E_{\text {consumed }}$ & Energy consumed in each activity \\
\hline$E_{\text {Total }}$ & Total amount of enrgy \\
\hline$r$ & Radio range of sensor node \\
\hline$R$ & Radio range of Base Station \\
\hline
\end{tabular}

\section{Algorithm for Selection of Aggregation Head}

Step $1 \quad$ Let sensor node i detects an event

Step $2 \quad$ Check if $\left(E_{S N \text { residual }}^{i}>E_{S N \text { min }}^{i}\right)$

2.1 If yes, then sensor node $\mathrm{i}$ sets itself to be the $\mathrm{AH}$ $(A H=$ true $)$ and sends an aggregation request message $(A g R q)$ to its neighbors $(N)$. The $A g R q$ message contains the residual energy $E_{A H \text { residual }}$, the distance to the base station distance $\boldsymbol{d}_{A H_{-} \text {to } B S}$ and the sensor node identifier SN_id.

Step 3 On receiving an $A g R q$ message from a sensor node $\mathrm{i}$, the sensor node $j$ checks if it has detected the same event.

3.1 If yes, it calculates its participation relevance $\mathrm{R}$ by given eq(). Check if $\left(R_{S N}>R_{\text {Threshold }}\right)$

3.1.a If yes, it decides to participate in aggregation and determines $A H$ ( $A H=$ true) only after checking $\left(E_{S N \text { residual }}>E_{A H \text { residual }}\right)$ and $\left(E_{S N \text { residual }}>E_{S N \text { min }}\right)$;

3.1.b If yes, then it compares distance to reach the base station $\left(\boldsymbol{d}_{S N_{-} \text {to } B S}<\boldsymbol{d}_{A H_{-} \text {to } B S}\right)$ and finally the identifiers.

Identifiers are used exclusively when two or more sensor nodes have the same residual energy and are at same distance from the station.

3.2 Otherwise, it will not participate in aggregation session. 


\section{Message Sending Algorithm}

If the sensor node is not an $A H$ then it will calculate its participation relevance. Participation relevance is determined by the decision metric presented in literature [10] to let the sensor nodes decide if they want to participate in the data aggregation session or not. This decision metric called the participation relevance $R_{P}$ in a data aggregation session is computed as

$$
R=\alpha \times \varepsilon+\beta \times \frac{1}{D}+\theta \times P+\omega \times I+C
$$

where

$\varepsilon$ is the energy efficiency,

$D$ is the density,

$P$ is the criticality of the node's position,

$I$ is the importance of the information.

$C$ is the approximation of constant.

$\alpha, \beta, \theta$ and $\omega$ are weighing factors,

Subjected to:

$\alpha+\beta+\theta+\omega+C=1$ and $0<(\alpha, \beta, \theta, \omega, C)<1$

The importance factors [10] of these parameters $\alpha, \beta, \theta, \omega$ and Care fixed to $0.25,0.10,0.25,0.25$ and 0.15 respectively. The constant $\mathrm{C}$ is a random variable between 0 and 0.15 . These values reflect the importance of their correspondent parameters. Based on these factors and through several simulations, we have found that the majority of values for participation relevance $R$ lie between 0.6 and 0.8 . Thus, we have chosen to fix the value of $R_{\text {Threshold }}$ to 0.7 .

Once $\mathrm{R}_{\mathrm{SN}}$ for each sensor node receiving the aggregation request is calculated, it is compared with $R_{\text {Threshold. }}$ If $R_{S N}>R_{\text {Threshold }}$ then the corresponding sensor node sends the aggregation reply message to selected $A H$ else not do anything. When the timeout occurs then $A H$ aggregate the received data from the participating sensor nodes and send concise message digest to the base station.

\section{E. Energy Consumption Computation Model}

We consider same radio model which has been used in [11] and shown in Fig. 1. If the distance between the transmitter and the receiver is less than a threshold $\left(d_{0}\right)$, the free space $\left(f_{s}\right)$ loss model is used; otherwise, the multi-path ( $\mathrm{mp}$ ) loss model is used [12]. The consumed amplifier energy, $E_{\text {amp }}$, of a sensor node is $E_{f s} . d^{2}$ or $E_{m p} . d^{4}$ depending on the distance $d$ between the sensor node and base station along with the acceptable bit-error rate [12]. It is given by

$$
E_{a m p}= \begin{cases}E_{f s} \cdot d^{2}, & \text { if } d<\mathrm{d}_{0} \\ E_{m p} \cdot d^{4}, & \text { if } d \geq \mathrm{d}_{0}\end{cases}
$$

Transmission power is adapted based on the distance to intend receiver and the threshold distance $d_{0}$ for substitution of amplification model. $d_{0}$ can be calculated by equating

$$
\begin{aligned}
E_{f s} \cdot d^{2} & =E_{m p} \cdot d^{4} \\
\mathrm{~d}_{0} & =\sqrt{\frac{E_{f s}}{E_{m p}}}
\end{aligned}
$$

Substituting typical values of $E_{f s}=10 \mathrm{pJ} / \mathrm{bit} / \mathrm{m}^{2}$

$$
\text { and } E_{m p}=1.3 \times 10^{-15} \mathrm{~J} / \mathrm{bit} / \mathrm{m}^{4} \text {, }
$$

$$
d_{0}=87.7 \mathrm{~m}
$$

If a sensor node transmits $b$ number of bits, the energy used in transmission will be

$$
\begin{gathered}
E_{T}(b, d)=E_{T-\text { elec }}(b)+E_{\text {amp }}(b, d) \\
E_{T}(b, d)= \begin{cases}b \cdot E_{\text {elec }}+b \cdot E_{f s} d^{2}, & \text { ifd }<\mathrm{d}_{0} \\
b . E_{\text {elec }}+b \cdot E_{m p} \cdot d^{4}, & \text { if } d \geq \mathrm{d}_{0}\end{cases}
\end{gathered}
$$

Similarly, to receive $b$ message bits, the sensor node spends

$$
E_{R}(b)=b . E_{\text {elec }}
$$

where $E_{\text {elec }}$ is energy in $\mathrm{J} / \mathrm{bit}$ for transmission and reception [13].

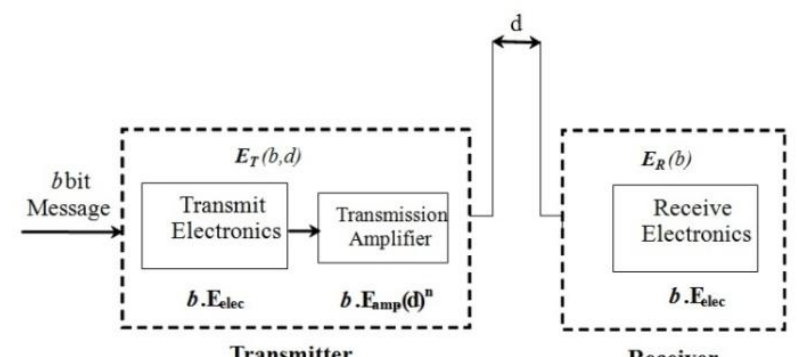

Fig. 1. Sensor radio model.

In this section, we describe the energy consumption model of proposed approach for aggregation head selection and message sending process. For simplicity we assumed that ' $n$ ' sensors are deployed in a circular region of radius $\mathrm{R}$, with base station located at the center of this region. We divide the region into two tiers of radius $r 1$ and $r 2$, so as to designate the distributed sensor nodes in normal, critical and edge positions as shown in Table II.

\begin{tabular}{lc} 
TABLE II: NORMAL, CRITICAL AND EDGE POSITIONS \\
\hline \hline Position & Region with radius \\
\hline Normal & $0<d<R$ \\
Critical & $25 \sqrt{2}<r 1<30 \sqrt{2}$ \\
Edge & $40 \sqrt{2}<r 2$ \\
\hline \hline
\end{tabular}

The energy consumption of the sensor node participating in the effective technique for data aggregation includes the energy to produce the neighborhood sensed data, the energy to receive $A g R q$ message, the energy to calculate participation relevance, the energy to reply and transfer sensed data. The energy consumption $E_{A}$ by the aggregation process at the initial node $i$ that detects the event, is given by:

$$
E_{A}^{i}=E_{A g R q}+N_{i} \times E_{A g R q R}+\sum_{i=1}^{N}\left(d_{i} \times E_{T}\right)+\sum_{i=1}^{k}\left(d_{i} \times E_{R}\right)
$$

where

$E_{A g R q}$ is energy consumed in sending the message by sensor node $i$.

$E_{A g R q R}$ is energy consumed in receiving the message by $N$ neighbor sensor nodes, 
$d_{i}$ is the distance between node $i$ and its $N$ neighboring sensor nodes,

$E_{T}$ is the energy consumed by transmission of each response message by $N$ neighboring sensor nodes,

$E_{R}$ is the energy consumed by the sensor node $i$ when it receives message from $k$ sensor nodes.

Total Aggregation Energy

$$
E_{A}=E_{A}{ }^{i}+\sum E_{A}^{i-1}
$$

Total Energy consumption in message transmission to base station, $E_{T R}$ is given by

$$
E_{T R}=\sum\left(d \times E_{T}\right)+\sum\left(d \times E_{R}\right)
$$

Total energy consumption $E$ using aggregation mechanism is given by:

$$
E=E_{T R}+E_{A}
$$

\section{Performance Evaluation}

In order to evaluate the relevance of our proposal, we have carried out a set of simulation tests. In this section, we define the simulation parameters in order to demonstrate the performance of our proposal by comparing it to Boughami $e$. $a l$, which appears to be an interesting approach. We have implemented these approaches on Matlab R2013 which is a scalable simulation environment for wireless network systems. In our simulation setup, as presented in Table III, we resume the different simulation parameters that we have used during the evaluation of our proposed model. We have evaluated the performance of suggested protocol through extensive simulations. In the simulation set up, the wireless sensor nodes are randomly deployed in a $100 \mathrm{~m} \times 100 \mathrm{~m}$ field with a base station located at centre (50, 50). Since transmission is single hop, communication between wireless sensor nodes and base station can take place only if the distance between them is within the transmission range.

\begin{tabular}{ll}
\multicolumn{2}{c}{ TABLE III: BASIC SIMULATION PARAMETERS } \\
\hline \hline PARAMETER & VALUE \\
\hline Area & $100 \mathrm{~m} * 100 \mathrm{~m}$ \\
No. of sensor nodes, $N$ & 1000 \\
Transmission Range & $30 \mathrm{~m}$ \\
Simulation Time & $20 \mathrm{minutes}$ \\
Data Rate & $250 \mathrm{kbps}$ \\
Energy Model & MicaZMote \\
Battery Model & $1200 \mathrm{mAhr}$ \\
Initial energy, $E_{\text {initial }}$ & $2 \mathrm{~J}$ \\
Energy consumed by radio electronics, Eelec & $50 \mathrm{~nJ} / \mathrm{bit}$ \\
Energy consumed by power amplifier, Eamp & $0.0013 \mathrm{pJ} / \mathrm{bit} / \mathrm{m}^{4}$ \\
Energy consumed by power amplifier, EFs & $10 \mathrm{pJ} / \mathrm{bit} / \mathrm{m}^{2}$ \\
Sensing range & $50 \mathrm{~m}$ \\
Initial network energy & $100 \mathrm{~J}$ \\
Transmission bit rate & $40 \mathrm{kbps}$ \\
Event occurrence frequency & $10 \mathrm{~s}$ \\
Receive power & $13.5 \mathrm{~mW}$ \\
Transmit power & $24.75 \mathrm{~mW}$ \\
Sleep power & $15 \mathrm{~W}$ \\
Idle power & $13.5 \mathrm{~mW}$ \\
\hline \hline
\end{tabular}

This shows that the proposed algorithm has more network lifetime than the old algorithm because in old algorithm the aggregation head selected on the basis of its residual energy only and this possibility may occur that a node that has highest energy can't be able to send the data to the sink due to the unknown distance from other nodes.

The network becomes dead at this time because it will choose the node as an aggregation head that is unable to send the data to the base station as evident from Fig. 2.Whether there can be a chance that the sensor node that has lower residual energy would be able to send data to the base station and this process increase the lifetime of the network, that is used in the proposed algorithm that select that node as the aggregation head that has lower residual energy but able to send data to the base station, this increase the total lifetime of a network.

The simulation result shows that the proposed algorithm provides 5-9\% more lifetime than the old algorithm as shown in Fig. 3.

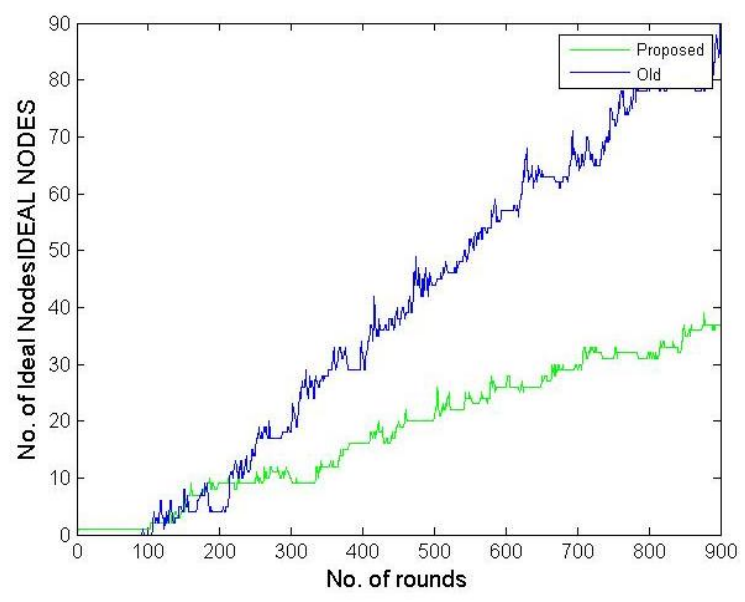

Fig. 2. Network lifetime with respect to no. of dead nodes.

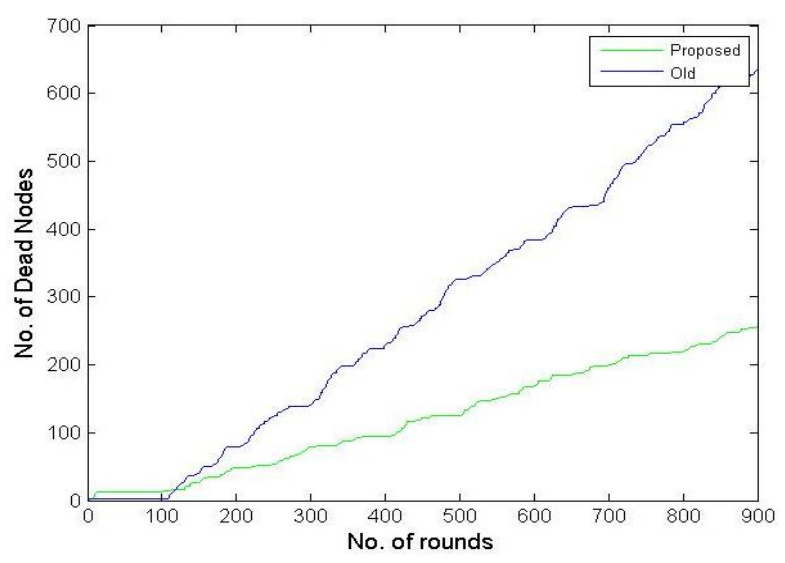

Fig. 3. Total energy consumption.

\section{CONCLUSION AND FUTURE WORK}

In this work, we proposed a data aggregation mechanism with selection of Aggregation Head on $E_{\min }, E_{\text {Res }}$ and nearness to base station. Simulation results show that this mechanism leads to enhancement of the sensor network performance. This protocol increases the lifetime of the network, selects that node as the aggregation head that has lower residual energy but able to send data to the base station, resulting the total lifetime of a network. 


\section{REFERENCES}

[1] F. Akyildiz, W. Su, Y. Sankarasubramaniam, and E. Cayirci, "A survey on sensor networks," IEEE Communications Magazine, vol. 40, no. 8, pp. 102-114, Aug. 2002.

[1] R. Rajagopalan and P. K. Varshney, "Data aggregation techniques in sensor networks: A survey," Electrical Engineering and Computer Science, p. 22, 2006.

[2] T. Koskiahde, J. Kujala, T. Norolampi, and F. Oy, “A sensor network architecture for military and crisis management," in Proc. International IEEE Symposium on Precision clock Synchronization for Measurement, Control and Communication, September 2008, pp. $110-114$.

[3] K. W. Fan, S. Liu, and P. Sinha, "Structure-free data aggregation in sensor networks," IEEE Transactions on Mobile Computing, vol. 6, no. 8, pp. 929-942, Aug. 2007.

[4] C. M. Chao and T. Y. Hsiao, "Design of structure-free and energy-balanced data aggregation in wireless sensor networks," in Proc. 11th IEEE International Conference on High Performance Computing and Communications, 2009, pp. 222-229.

[5] A. Sardouk, M. Mansouri, L. Merghem-Boulahia, D. Gaiti, and R. Rahim-Amoud, "Multi-agent system based wireless sensor network for crisis management," in Proc. 2010 IEEE Global Telecommunications Conference, 2010, pp. 1-6.

[6] N. Boughanmi, M. Esseghir, L. Merghem-Boulahia, and L. Khoukhi, "Energy efficient aggregation in wireless sensor networks," in Proc. ICAIT 2012, 2012, pp. 264-273.

[7] A. Sardouk, R. Rahim-Amoud, L. Merghem-Boulahia, and D. Gaiti, "A Multi-criteria data aggregation scheme for WSN," in Proc. WiMob. Marrakech, 2009.

[8] S. Yadav and R. S. Yadav, "A review on energy efficient protocols in wireless sensor networks," Wireless Networks, vol. 2, no. 6, 2015.

[9] A. Sardouk, R. Rahim-Amoud, L. Merghem-Boulahia, and D. Gaiti, "A strategy for multi-agent based wireless sensor network optimization," Scalability of Networks and Services, pp. 122-133, 2009.
[10] W. R. Heinzelman, A. Chandrakasan, and H. Balakrishnan, "An application specific protocol architecture for wireless microsensor networks," IEEE Transactions on Wireless Communications, vol. 1, no. 4, pp. 660-670, 2002.

[11] K. Sohraby, D. Minoli, and T. Znati, Wireless Sensor Networks, Technology, Protocols, and Applications, New York, Willey, 2007.

[12] T. Rappaport, Wireless Communications: Principles Practice, Englewood Cliffs, New Jersey: Prentice-Hall, 1996.

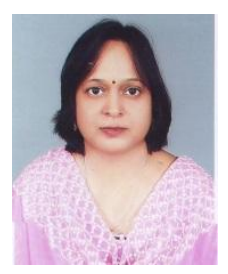

Sarika Yadav is presently pursuing a Ph.D. degree from the Motilal Nehru National Institute of Technology (MNNIT), Allahabad, India. She received her M.Tech. degree in computer science and engineering from Uttar Pradesh Technical University, Lucknow. Her research interests are in the field of fault tolerance systems, wireless sensor networks and artificial intelligence.

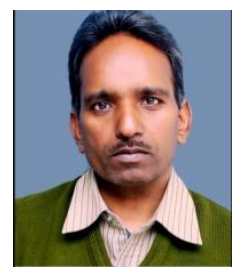

Rama Shankar Yadav is currently a professor at Motilal Nehru National Institute of Technology, Allahabad, India. He received his Ph.D. degree from the Indian Institute of Technology (IIT), M.S. degree from Birla Institute of Technology and Science (BITS) Pilani, and B.Tech. degree from the Institute of Engineering and Technology (I.E.T.), Lucknow, India. Dr. Yadav has extensive research and academic experience. He has worked in leading institutions such as Govind Ballabh Pant Engineering College (GBPEC), Pauri, Garhwal, and Birla Technical Training Institute (BTTI), Pilani. He has authored more than 45 research papers in national/international conferences, refereed journals, and book chapters. Dr. Yadav's areas of interest are real time systems, embedded systems, fault-tolerant systems, energy aware scheduling, network survivability, computer architecture, distributed computing, and cryptography. 